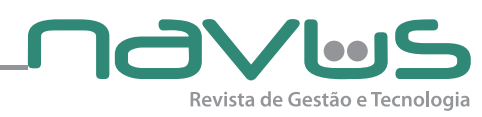

\title{
ULBRATECH: desafios e benefícios na implantação de uma rede de inovação
}

\author{
Márcio Roberto Machado da Silva ${ }^{1}$ \\ Anderson Ricardo Yanzer Cabral ${ }^{2}$ \\ Eduardo Basso Junior ${ }^{3}$ \\ Ariel Dutra Birnkott ${ }^{4}$
}

\section{RESUMO}

Este artigo apresenta a experiência do desenvolvimento de uma rede de inovação, a Rede de Inovação ULBRATECH, na forma de experiências e lições aprendidas, obtidas por meio do planejamento e da implantação da referida rede, que compreende incubadoras e parques tecnológicos, e núcleos ou agências de inovação e transferência de tecnologia, em nível nacional, no Brasil. A rede está organizada em quinze cidades brasileiras, compreendendo seis estados das regiões sul, centro-oeste e norte do Brasil, onde a universidade mantenedora atua com unidades de ensino superior. Foi realizada uma pesquisa exploratória de caráter qualitativo com os gestores dos empreendimentos de inovação e gestores das unidades de ensino da universidade, e identificaram-se os principais desafios e dificuldades da estruturação da rede, assim como as principais vantagens obtidas por essa rede. Os resultados são apresentados no formato de lições aprendidas e servem como uma fonte de conhecimento para instituições que pretendem implantar redes de inovação. Dentre os principais resultados, destacam-se a dificuldade de integração dos modelos de gestão da universidade e dos empreendimentos de inovação, em razão das diversidades regionais; problemas de gestão do conhecimento, que impactam na integração entre os partícipes da rede, tanto em virtude da falta de compartilhamento das experiências, como a dificuldade de manter um diretório atualizado de competências da rede; necessidade de fomentar a cultura empreendedora de forma interna e externa à universidade; e a necessidade de revisão de processos de gestão da rede, com vistas a qualificar o modelo de governança da Rede de Inovação.

Palavras-chave: Redes de inovação. Parques tecnológicos. Incubadoras tecnológicas.

\section{INTRODUÇÃO}

Os habitats de inovação têm se apresentado como elementos importantes no desenvolvimento de projetos, tanto nas universidades quanto nas empresas e no setor governamental, que buscam inovação.

O diferencial dos projetos de incubadoras e parques tecnológicos da Universidade Luterana do Brasil (ULBRA) está relacionado ao fato de esses projetos trabalharem em rede, utilizando-se da estrutura da rede de ensino da ULBRA, que compreende quinze unidades espalhadas pelo Brasil, para desenvolver

\footnotetext{
1 Mestre - Universidade Luterana do Brasil - marcio.machado@ulbra.br

${ }^{2}$ Doutor - Universidade Luterana do Brasil - anderson.yanzer@ulbra.edu.br

${ }^{3}$ Mestre - Universidade Luterana do Brasil - eduardobasso@gmail.com

${ }^{4}$ Especialista - Universidade Luterana do Brasil - ariel@ulbratech.com.br
} 
pesquisa, desenvolvimento e inovação. Como suporte à implantação dos "nós" da Rede ULBRA de Inovação (ULBRATECH), destacam-se duas atividades desenvolvidas: visitações e ações decorrentes destas, e realização de entrevistas com os gestores das unidades de ensino e inovação da universidade.

Este artigo teve como objetivo explorar a percepção dos integrantes da ULBRATECH quanto à sua implantação e desenvolvimento. A rede em questão está presente em quinze unidades da ULBRA em todo o Brasil, sendo cada unidade compreendida como um "nó" da rede.

Dessa forma, este artigo apresenta inicialmente um referencial teórico que aborda conceitos de alguns tipos de habitats de inovação. Na sequência, é apresentada a ULBRATECH, que serviu de contexto para este texto. Em seguida, é apresentado o método de pesquisa no qual foi desenvolvido o trabalho, seguido da descrição da metodologia de implantação desta rede, da análise dos dados coletados, que foi a base para elaboração da próxima seção, as lições aprendidas. Por fim, são apresentadas as conclusões e o referencial bibliográfico.

\section{OS PARQUES TECNOLÓGICOS E AS INCUBADORAS}

De acordo com Clark (2004), no último quarto do século XX, as universidades sofreram grande pressão para mudar a maneira como operavam. Segundo o autor, as universidades precisaram desenvolver a capacidade de flexibilidade em seus ambientes para combinar o velho e o novo, a mudança e a continuidade.

Os incubadores de empresas e parques tecnológicos, segundo Vedovello (2000), também conhecidos como habitats de inovação, fazem parte de um mesmo grupo de ferramentas de política tecnológica e industrial, com pontos em comum, como agrupamento de empresas que apresentam como um de seus diferenciais a proximidade de outras empresas e instituições, notadamente as universidades, com grandes possibilidades de sinergias. Ao mesmo tempo, segundo Du Plessis (2007), a inovação é dependente em grande medida da disponibilidade de conhecimento e isso implica um gerenciamento por meio de práticas de gestão do conhecimento, como uma forma de garantir o sucesso da inovação.

Apesar de apresentarem algumas características comuns, os Parques Tecnológicos e Incubadoras de Empresas são organizações com particularidades que os diferenciam; porém, como um dos pontos mais importantes, ao mesmo tempo, compartilham um atributo. Parques tecnológicos e incubadoras de empresas, segundo a Associação Nacional de Entidades Promotoras de Empreendimentos Inovadores (ANPROTEC), apresentam-se como organizações promotoras de empreendimentos que visão a inovação (ANPROTEC, 2013). Esse processo, no entanto, demanda instrumentos de administração que, conforme Lipparini e Sobrebro (1997), são os ajustes mútuos entre os participantes, por meio de decisões conjuntas; a realização da coordenação e acertos das atividades da rede por alguém que atue como mediador e facilitador das relações na rede; a administração por uma autoridade central; e a institucionalização de normas e obrigações para controle entre as partes e com outros atores. Por outro lado, é importante ter um ambiente de cooperação e colaboração para o sucesso das redes de inovação (PELLEGRIN et al, 2007).

A incubadora de empresas é um espaço empresarial compartilhado que procura proporcionar aos seus incubados um sistema estratégico de intervenção com agregação de valor, de monitoramento e assistência nos negócios. (HACKETT; DILTS, 2004).

Segundo a Associação Nacional das Incubadoras de Empresas dos Estados Unidos da América (NBIA), a incubação de empresas é um processo de apoio às empresas que acelera o desenvolvimento de empresas startups e inexperientes, fornecendo aos empreendedores um conjunto de recursos e serviços direcionados. Esses serviços são geralmente desenvolvidos ou orquestrados por gestão da incubadora e são oferecidos tanto na incubadora de empresas quanto pela sua rede de contatos. A meta principal da incubadora de empresas é a produção de empresas de sucesso.

As empresas graduadas têm o potencial de criar empregos, revitalizar bairros, comercializar novas tecnologias e fortalecer a economia local e nacional. Para a associação, o fundamental na definição de uma incubadora é o fornecimento de orientações de gestão, assistência técnica e consultoria sob medida para empresas jovens e em crescimento. Incubadoras geralmente também oferecem aos clientes o acesso ao espaço apropriado, aluguel flexível, serviços empresariais básicos compartilhados e equipamentos, serviços de suporte tecnológico e assistência na obtenção do financiamento necessário para o crescimento da empresa (NBIA, 2013).

A incubadora de empresas tem a finalidade de apoiar empreendedores a se tornarem empresas bemsucedidas, incentivando o desenvolvimento de ideias inovadoras e oferecendo infraestrutura e suporte gerencial, para o desenvolvimento dos negócios e da gestão do empreendedor (ANPROTEC, 2013).

Incubadora é um centro que abriga, habitualmente, microempresas de base tecnológica em um mesmo espaço físico, dividido em módulos e próximo às Instituições de Ensino e Pesquisa, para poder utilizar dos 
seus recursos humanos e materiais (MEDEIROS et al, 1992).

Os parques tecnológicos compõem uma aglomeração de empresas de base tecnológica, conectadas a universidades e centros de pesquisa e desenvolvimento (P\&D), possibilitando sinergias entre os atores (VEDOVELLO, 2000).

Os Parques Tecnológicos são

um complexo produtivo industrial e de serviços de base científico-tecnológica. Planejados, têm caráter formal, concentrado e cooperativo, agregando empresas cuja produção se baseia em Pesquisa e Desenvolvimento (P\&D). Assim, os parques atuam como promotores da cultura da inovação, da competitividade e da capacitação empresarial, fundamentados na transferência de conhecimento e tecnologia, com o objetivo de incrementar a produção de riqueza de uma determinada região (ANPROTEC, 2013).

Já a United Kingdom Science Park Association (UKSPA) define um parque tecnológico como uma iniciativa de apoio às empresas e transferência de tecnologia que (a) estimula e apoia o início e incubação de negócios de inovação, com alto crescimento, baseados em conhecimento; (b) fornece um ambiente onde até empresas grandes e internacionais podem desenvolver interações específicas e próximas com um determinado centro de criação de conhecimento para um benefício mútuo; (c) tem ligações formais e operacionais com centros de criação de conhecimento, tais como universidades, institutos de ensino superior e organizações de pesquisa (UKSPA, 2013).

Espera-se que um parque tecnológico aumente a transferência de conhecimento entre universidade e empresa, para gerar novos negócios e desenvolver os já existentes, e, além disso, atraia o desenvolvimento para toda a região em seu entorno.

De acordo com Medeiros et al (1992), em um parque tecnológico, as empresas agrupadas em um local muito próximo à Instituição de Ensino e Pesquisa (IEP) possuem uma instituição que coordena e que gerencia os recursos e promove a interação entre as empresas e a IEP. Nesse ambiente, os principais recursos compartilhados são fundamentalmente os suportes técnico-científicos; por isso, a importância da proximidade das IEPs.

Tanto os parques tecnológicos como as incubadoras de base tecnológica, para terem sucesso, dependem da presença de IEPs de alto nível na região, assim como, de um ambiente econômico dinâmico, com potencial de instigar o empreendedorismo.

O Brasil possui 384 incubadoras em operação, que abrigam 2.640 empresas, gerando 16.394 postos de trabalho. No país, 2.509 empreendimentos já foram graduados, faturam $\mathrm{R} \$ 4,1$ bilhões e geram 29.205 empregos. Outro dado relevante é que $98 \%$ das empresas incubadas inovam; dessas, $28 \%$ têm foco local, $55 \%$ nacional e 15\% mundial, segundo estudo realizado em 2011 pela ANPROTEC, juntamente com o Ministério de Ciência, Tecnologia e Inovação (MCTI) (ANPROTEC, 2013).

Quando se procura na literatura por redes de inovação, normalmente as referências apontam para ambientes de colaboração e inteligência coletiva, como pode ser observado em Gloor (2005). 0 contexto do conceito de rede de inovação apresentado neste trabalho está orientado para uma estrutura de rede composta por parques e incubadoras tecnológicas, que colaboram de forma a obter vantagens competitivas no compartilhamento de recursos e serviços.

\section{ULBRATECH - A REDE ULBRA DE INOVAÇÃO}

A Rede ULBRA de Inovação é uma rede que integra os projetos de parques tecnológicos, incubadoras tecnológicas, núcleo de inovação e transferência tecnológica (NITT) e convênios de cooperação científica e tecnológica entre a ULBRA e outras instituições nacionais e internacionais, formando uma grande rede de cooperação que objetiva o desenvolvimento de pesquisa, desenvolvimento e inovação entre as partes envolvidas, utilizando-se de estrutura compartilhada, tanto de laboratórios quanto de pessoal técnico e administrativo, assim como as sub-redes mantidas pelos partícipes do processo.

Essa rede de inovação já nasce com o potencial de presença da rede de ensino da universidade, nas cidades onde a ULBRA mantém unidades de ensino superior presencial. A ULBRA está presente em nove cidades no Rio Grande do Sul: Canoas, Porto Alegre, Gravataí, Guaíba, Torres, São Jerônimo, Carazinho, Cachoeira do Sul e Santa Maria; e em mais seis cidades em outros estados: Palmas/TO, Itumbiara/GO, Porto Velho e Jí-Paraná/RO, Santarém/PA e Manaus/AM. 
No primeiro ano de implantação do projeto da Rede ULBRA de Inovação, já está presente, por meio de Incubadora Tecnológica, Parque Tecnológico ou NITT, em sete dessas cidades.

Cabe salientar, como diferencial, que a Rede ULBRA de Inovação, totalmente integrada com a Universidade, conta com uma estrutura técnica de apoio altamente qualificada em virtude de cursos específicos de graduação (cursos de bacharelado, tecnológicos e de licenciatura) e pós-graduação (especialização, mestrado e doutorado), que oferecem estrutura de apoio, tanto em termos de corpo técnico / docente como de pesquisas.

\subsection{Estrutura da rede ULBRA de inovação}

A ULBRA é a organização mantenedora da ULBRATECH, sendo que possui diversos cursos e centros de excelência de pesquisa em diversas áreas. Tem, por meio do NITT, a preocupação de unir ensino, pesquisa e extensão através de seus profissionais com o intuito de gerar inovação no ambiente da ULBRATECH. Esta, por sua vez, se vale destas iniciativas para contribuir para as organizações instaladas em sua rede, integrando-as com profissionais de cursos de graduação e pós-graduação.

A ULBRATECH está diretamente vinculada à Diretoria de Inovação, ligada à Pró-reitoria de PósGraduação, Pesquisa e Inovação da ULBRA, sob a gestão e responsabilidade da Diretoria de Inovação, tendo sua estrutura de governança representada na Figura 1.

A Diretoria de Inovação tem à disposição, além das estruturas dos Parques Tecnológicos, das Incubadoras Tecnológicas e dos Comitês de Inovação de cada campi, o NITT. O NITT possui um papel fundamental no fomento à produção, registro e manutenção da propriedade intelectual da Rede ULBRA de Inovação, oferecendo suporte jurídico e técnico para todo o corpo de pesquisadores e para as empresas da rede.

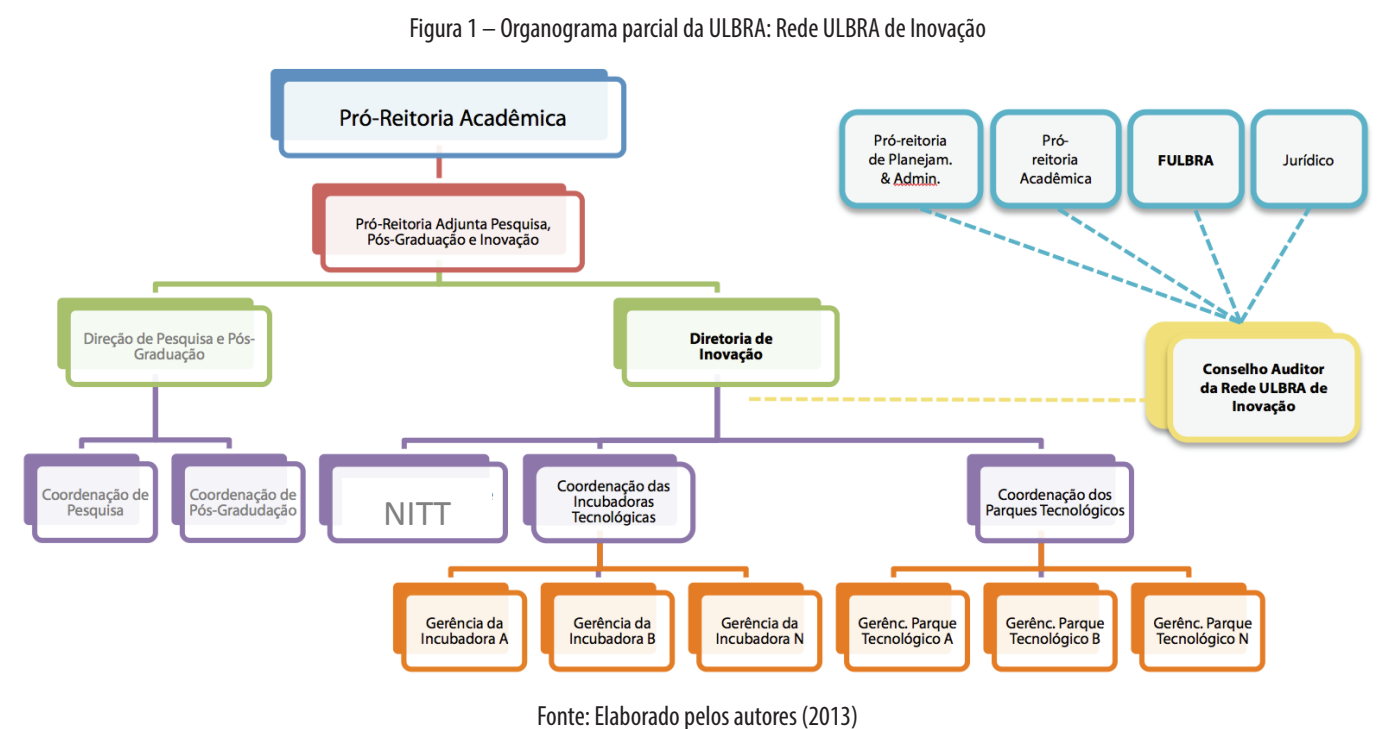

Como observado na Figura 1, a Diretoria de Inovação é a responsável institucional do projeto da Rede ULBRA de Inovação, que contempla as iniciativas de incubadoras tecnológicas e parques tecnológicos no Brasil, além de definir políticas e propor ações relacionadas à inovação, tanto em âmbito acadêmico quanto administrativo.

Para a execução das competências e atribuições da Diretoria de Inovação, esta é composta pela seguinte estrutura: (a) Coordenação dos Parques Tecnológicos; (b) Coordenação das Incubadoras Tecnológicas; (c) Coordenação dos Núcleos de Inovação e Transferência Tecnológica; e (d) Comitês de Inovação. A coordenação dos parques tecnológicos, ligada à Diretoria de Inovação, é a responsável pela articulação dos parques tecnológicos em todas as unidades da ULBRATECH, desde o planejamento, implantação e gestão, assim como é a facilitadora da integração deles.

A coordenação dos Parques Tecnológicos está subordinada à Diretoria de Inovação, sendo responsável pela gestão dos parques em todas as unidades da ULBRATECH, estabelecendo as políticas gerais para todos os parques integrantes da rede. Integrando a estrutura da coordenação dos Parques Tecnológicos tem as 
gerências dos Parques Tecnológicos da ULBRATECH, diretamente ligadas à direção geral da unidade, as quais são as responsáveis pela gestão executiva do parque tecnológico em cada unidade da ULBRA.

A coordenação das Incubadoras Tecnológicas, ligada à Diretoria de Inovação, é a responsável pela articulação das incubadoras tecnológicas em todas as unidades da ULBRATECH, desde o planejamento, implantação e gestão, assim como facilitar a integração destas. Já as gerências dos Parques Tecnológicos da ULBRATECH, diretamente ligadas à direção geral da unidade, são as responsáveis pela gestão executiva dos parques nas unidades da ULBRA.

O Comitê de Inovação, em cada unidade da ULBRA, ligado à Direção Geral da Unidade e à Direção de Inovação da ULBRA, é o órgão colegiado assessor dos gestores do parque tecnológico e da incubadora tecnológica, tanto para questões de seleção e avaliação dos projetos das empresas da ULBRATECH como para acompanhamento das ações ligadas à inovação.

O NITT é o responsável pelo fomento e gestão da propriedade intelectual desenvolvida em todos os setores da universidade, desde a graduação, pós-graduação e pesquisa, até os parques tecnológicos e as incubadoras. Cabe, ainda, ao NITT, o gerenciamento dos contratos e convênios que envolvam inovação e transferência tecnológica na universidade.

\section{MÉTODO DE PESQUISA}

Esta pesquisa de caráter exploratório e abordagem qualitativa teve como objetivo diagnosticar a percepção dos integrantes dos habitats de inovação da ULBRATECH em todo o Brasil. Foi um estudo de caso que, segundo Yin (2010, p. 39), é "uma investigação empírica que investiga um fenômeno contemporâneo em profundidade e em seu contexto de vida real". Além disso, no estudo de caso, busca-se responder "como" e "por quê", questões estas que "lidam com vínculos operacionais que necessitam ser traçados ao longo do tempo, mais do que as meras frequências ou incidências" (YIN, 2010, p. 30).

A coleta dos dados foi feita mediante roteiro de entrevista semiestruturado e por observações in loco. A coleta ocorreu com os integrantes da ULBRATECH, tanto no habitat dos Parques Tecnológicos como no das Incubadoras Tecnológicas. Participaram da pesquisa os envolvidos no processo de gestão da ULBRATECH, sendo a coleta feita com vinte dos trinta integrantes.

0 roteiro de entrevista teve como objetivo diagnosticar a percepção dos integrantes da ULBRATECH quanto à situação da instituição em sua unidade e suas perspectivas. Além disso, foi utilizada a observação assistemática, que, segundo Michel (2009, p. 66), é "não estruturada, espontânea, informal, ordinária, simples, livre, ocasional". Essas observações ocorreram ao longo de 2012 e parte de 2013 pela equipe da Diretoria de Inovação da ULBRATECH.

Quanto à análise dos dados, optou-se por agrupar as respostas por convergências e divergências de ideias (YIN, 2010) e, posteriormente, organizá-las em lições aprendidas.

\section{O CASO DA REDE DE INOVAÇÃO ULBRATECH}

Nesta seção, são apresentados o processo de criação de um "nó" da rede ULBRATECH e as duas principais ações que caracterizam os passos mais importantes e que servem de base para a implantação da rede.

Dentro desse processo de implantação, a demanda de criação de um "nó" da rede ULBRA de inovação nasce da direção da unidade (campus) da universidade, que entra em contato com a Diretoria de Inovação e manifesta seu interesse em ingressar no projeto, caracterizando uma demanda regional. A partir daí, agenda-se a primeira visita in loco para conhecer a estrutura do campus em questão e para a apresentação do projeto da Rede ULBRA de Inovação para a equipe diretiva da referida unidade.

Sugere-se que sejam realizadas reuniões com o poder público, principalmente com secretarias relacionadas à inovação. Estas reuniões normalmente acontecem com as secretarias estaduais de ciência, tecnologia e desenvolvimento tecnológico, secretarias de desenvolvimento, de indústria e comércio, além de órgãos como Serviço Brasileiro de Apoio às Micro e Pequenas Empresas (SEBRAE) e da Fundação de Amparo à Pesquisa dos estados (FAPs). Também se recomenda encontros com as principais entidades de classe (empresariais), bem como com empresários e empreendedores de destaque na região, com o intuito de apresentar o projeto e que propicie a adesão ou apoio.

A Diretoria de Inovação desenvolveu um roteiro para essa visita, que contempla:

- Conhecimento da região (vocações regionais, potencialidades técnico-científicas, políticas de incentivos à inovação, disponibilidade de espaço físico e infraestrutura, potencialidades de parcerias com os 
setores público e privado).

- Definição do modelo/tipo do empreendimento (incubadora, parque, NITT ou ambos) e personalidade jurídica.

- Definição do gestor, comitê de inovação e estrutura de apoio/secretária (nomes e dedicação ao projeto)

- Definição de espaços físicos e infraestrutura mínima (móveis, equipamentos, telefonia, internet, elétrica, etc.)

- Definições das políticas de interação do empreendimento com o ensino, pesquisa e extensão da unidade (ensino, pesquisa, extensão, descontos e benefícios em geral).

- Definição da política de seleção das empresas e projetos e do edital de seleção.

- Definição da identificação visual.

- Adaptações do Projeto da Rede ULBRA de Inovação.

- Contrato de confidencialidade com os membros do Comitê de Inovação.

- Orientações para elaboração do Planejamento Estratégico e Plano de Marketing.

- Orientações sobre a implantação do Prêmio Projeto Inovador.

- Orientações sobre a necessidade de Capacitação no Modelo CERNE.

- Articulações Políticas.

- Criação da estrutura web: portal ULBRATECH (criar usuário e dar orientações iniciais), e-mails @ ulbratech.com.br e configuração do compartilhamento dos repositórios de documentos na nuvem.

- Orientações gerais a respeito das principais tarefas cotidianas, assim como dos tipos de documentos e seus arquivamentos.

Esse roteiro é enviado previamente para o diretor da unidade para que ele trabalhe questões internas, como a construção da equipe que atuará no projeto, subsídios sobre as vocações regionais e as competências técnicas, tanto de laboratórios quanto de pesquisadores e cursos de graduação e pós-graduação da unidade, até mesmo a articulação política para o projeto.

No fim de cada visita, é feito um relatório para registro de todas as atividades desenvolvidas, assim como para registro da percepção da diretoria de inovação para com o andamento do projeto, destacando pontos positivos e negativos. Esse relatório serve como ponto de partida para o planejamento estratégico do empreendimento no campus.

\section{ANÁLISE DOS DADOS}

Apresentamos a seguir as principais lições aprendidas neste projeto, que foram percebidas com base na experiência vivenciada pelos gestores da rede ULBRA de inovação no processo de implantação dos "nós" da rede e também na análise dos dados obtidos por meio dos instrumentos utilizados na pesquisa realizada com os gestores das unidades que compõem a rede de inovação ULBRATECH.

As lições aprendidas apresentadas apontam questões que se aplicam em um ambiente de rede; todavia, algumas delas podem ser aplicadas e observadas em habitats de inovação que trabalham com modelos individuais (fora de redes de inovação).

\subsection{O mito do sucesso pela inovação como mágica}

O sucesso que o termo "inovação" tem conquistado nas mídias nacionais e internacionais, sempre atrelado ao sucesso e à modernidade, juntamente com os diversos recursos financeiros oferecidos, normalmente pelo governo, para fomentar a inovação, tem feito com que diversas pessoas acreditem que a simples adoção do termo "inovação" aos seus negócios pode gerar sucesso. Mera miopia organizacional.

Alguns gestores, tanto governamentais quanto educacionais, por impulso das notícias de sucesso de grandes empreendimentos relacionados a Parques Tecnológicos, pelo movimento das grandes empresas, buscando instalação em ambientes de inovação, e por imaginar que um parque tecnológico é um simples espaço para inovação, têm oferecido espaço e estrutura física para instalação de novos parques tecnológicos.

Contudo, um dos trabalhos da equipe da diretoria de inovação da ULBRA foi a de desmitificar os parques tecnológicos. Mostrar para os gestores que, quando falamos em ambiente de inovação, não se resume a um espaço e infraestrutura física, predial, pronta para receber empresas.

Tem-se mostrado que um parque tecnológico é um ambiente de inovação altamente especializado, que necessita de um nível de maturidade por parte de todos os atores diretamente envolvidos no projeto: universidade ou instituto de ciência e tecnologia (ICT), governo e empresas. Não adianta somente um ou 
dois dos atores dessa tríplice hélice estarem maduros em relação a diversos e diferentes fatores, como cultura empreendedora, criatividade, pesquisas aplicadas, trabalho colaborativo, etc.

Assim, a primeira missão, ao chegar a uma nova cidade, é fazer um diagnóstico do nível cultural a fim de definir quais modelos podem ser implantados e desmitificar os conceitos de inovação e seus atores.

6.2 Dificuldade em ter modelos padrões para os empreendimentos da rede e a consequente necessidade de adaptação dos modelos de gestão em razão da cultura regional, organizacional, política e econômica

Vedovello, Judice e Maculan (2006) destacam a importância de, no processo de implementação de parques, existirem maneiras de provocar os ajustes necessários, como forma de torná-los os mais adequados possíveis à realidade na qual eles, efetivamente, operarão. Ou seja, que estejam ajustados à realidade regional.

Nas visitas, pode-se notar que alguns gestores têm ótimo relacionamento com o setor público, outros com o setor privado, e outros ainda estão construindo essa relação, ficando nesse momento focado no público interno.

Essas diversidades igualmente podem estar relacionadas à maturidade do governo para com o tema inovação. Percebeu-se uma diferença muito grande no nível de entendimento e de estrutura pública de suporte à inovação. Enquanto alguns estados, como o Rio Grande do Sul e Goiás, trabalham com políticas de incentivo à inovação que têm evoluído a aproximadamente dez anos, outros estados estão apenas iniciando um mapeamento de iniciativas de inovação.

E essa diferença atinge todos os atores da tríplice hélice (governo, empresas e universidade); além disso, observam-se grandes distinções em termos de incentivo e investimentos em inovação por parte dos empresários das cidades e estados visitados.

Essa diversidade dificulta que seja criado um modelo nacional de estruturação e trabalho das iniciativas da Rede ULBRA de Inovação, seja parque tecnológico, incubadora tecnológica ou NITT.

6.3 Do ponto de vista de gestão do conhecimento, detectaram-se a necessidade de definição de mecanismos de integração entre os partícipes da rede e a importância do mapeamento de competências e potencialidades técnicas e científicas tanto das empresas quanto, principalmente, da universidade

0 tema gestão do conhecimento tem sido foco de pesquisa pelos habitats de inovação, haja vista a necessidade de explorar e administrar o conhecimento como um fator gerador de inovação. Segundo Du Plessis (2007), a inovação é extremamente dependente da disponibilidade de conhecimento e, portanto, a complexidade criada pela explosão de riquezas e da quantidade de conhecimento tem de ser gerenciada, por intermédio de práticas de gestão do conhecimento, como uma forma de garantir o sucesso da inovação.

Nas reuniões que ocorrem durante as visitas de implantação do projeto ULBRATECH, principalmente as realizadas com empresários ou dirigentes de entidades de classe, constatou-se a necessidade de conhecer as competências e potencialidades técnicas e científicas dos professores da universidade, para que seja oferecido um pacote de possibilidades para o setor empresarial.

A universidade, normalmente, tem essa informação de competências científicas por meio dos currículos de seus professores e do cadastro de seus laboratórios. Contudo, notou-se que essa informação, da forma como está armazenada, no site do CNPq - Plataforma Lattes, é de difícil recuperação, e não representa a totalidade de oportunidades. Foram detectados diversos casos nos quais existe a competência, mas não está evidenciada pela pesquisa cadastrada no currículo do professor.

Nesse caso de mapeamento de competências, em se tratando de empresas, o problema é ainda maior, pois os colaboradores destas não têm o hábito de cadastrar seu currículo em um banco de dados como a plataforma Lattes do CNPq, e tampouco mantêm seus currículos atualizados.

Desse modo, sugere-se o uso de um sistema de informação para implementar um banco de competências e oportunidades, assim como organizar instrumentos de divulgação dos projetos, competências e oportunidades entre os partícipes da rede.

6.4 Necessidade de incentivar e trabalhar ativamente para o desenvolvimento de uma cultura empreendedora na região, tanto dentro quanto fora da universidade

De acordo com Clark (2004), uma universidade empreendedora atua como uma instituição ativa que faz mudanças na sua estrutura e no modo de reagir às demandas internas e externas, e aponta a cultura empreendedora integrada como fator crítico para o sucesso da mudança e para a geração de uma perspectiva 
institucional.

Diante dessa visão de empreendedorismo, percebeu-se, em algumas regiões, a necessidade de fomentar a cultura empreendedora, tanto dentro quanto fora da universidade, divulgando toda a estrutura de apoio ao empreendedor oferecida pela rede ULBRATECH e desmitificando o empreendedorismo.

Acredita-se que os cursos de graduação sejam grandes geradores de ideias que possam ser transformadas em negócios; entretanto, os alunos não são incentivados a desenvolver seus projetos de conclusão de curso com essa visão empreendedora.

Assim sendo, verifica-se uma dificuldade de integração da rede ULBRATECH com a academia por não haver um núcleo de inovação integrando todos os cursos de graduação e pós-graduação, que possa criar e coordenar disciplinas de inovação e empreendedorismo, assim como atuar como um facilitador para que o empreendedorismo seja um tema transversal a todas as disciplinas dos cursos da universidade.

\subsection{Possibilidade de cooperação entre as unidades da rede de inovação}

Pellegrin et al (2007) destacam a importância da cooperação e colaboração para o sucesso das redes de inovação e, já desde o início de projeto, pode-se evidenciar a efetiva cooperação das entidades que participam da rede ULBRATECH, demonstrando que o conceito da rede de inovação funciona e traz benefícios para os partícipes do projeto.

Trata-se do caso de uma empresa do Parque Tecnológico ULBRATECH Itumbiara/Goiás, que tinha um problema para ser resolvido, relacionado à destinação de resíduos da queima de sabugo de milho, que, depois de certo tempo, passou a ter características que impediam que fosse descartado na natureza.

Como o Parque Tecnológico ULBRATECH Itumbiara é focado em agronegócios, seus laboratórios são diretamente ligados a essa área, não tendo profissionais da área de materiais. Sendo assim, foi acionado o laboratório petroquímico do ULBRATECH Canoas, que se utilizando de seus equipamentos, como, por exemplo, analisador de partículas laser, conseguiu analisar o resíduo e apresentar uma variedade de opções para resolução do problema, inclusive algumas delas com possibilidade de geração de recursos pela venda desse resíduo tratado.

6.6 Necessidade de revisão e reestruturação do modelo de gestão e de alguns processos na universidade, para atender às demandas e velocidades exigidas pelo setor empresarial

Notou-se, neste primeiro ano do projeto, a existência de uma grande diferença entre os tempos e prazos decisórios administráveis pela universidade e pelas empresas.

A universidade trabalha em um ciclo semestral, com um planejamento de recursos semestral, em que a maioria das decisões são tomadas em um período que antecede o início do semestre e não são modificadas depois desse momento.

Contudo, diferentemente disso, as empresas são muito mais dinâmicas e acostumadas a resolver novos problemas todos os dias. Elas têm mecanismos de gestão que dão total autonomia ao empresário ou aos tomadores de decisão, o que possibilita maior agilidade de ação e reação.

Lipparini e Sobrero (1997) apresentam como principais instrumentos de administração das redes de inovação: (a) ajustes mútuos entre os participantes, mediante decisões conjuntas; (b) realização da coordenação e acertos das atividades da rede por alguém que atue como mediador e facilitador das relações na rede; (c) administração por uma autoridade central; e (d) institucionalização de normas e obrigações para controle entre as partes e com outros atores.

Dessa forma, a universidade deve revisar seu organograma funcional e modelo de gestão de modo a possibilitar a criação de estruturas e definição de processos que contemplem capacidade de decisão desvinculada do setor acadêmico, tanto pela diferença do ciclo de decisões como pela diferença das fontes de captação de recursos e das diversas necessidades de investimentos.

\section{CONCLUSÃO}

Este artigo apresentou a experiência de implantação da Rede ULBRA de Inovação ULBRATECH, que é composta por uma rede de parques e incubadoras tecnológicos e Núcleos de Inovação e Transferência de Tecnologia, distribuída em quinze cidades no Brasil, onde a Universidade Luterana do Brasil mantém unidades de ensino superior na modalidade presencial. 
As principais contribuições deste artigo foram apresentadas na forma de lições aprendidas, que sintetizam a experiência da implantação de ambientes de inovação, em rede, que foram obtidas por meio de observações diretas realizadas em visitas in loco às unidades da ULBRA e pelas entrevistas realizadas com os gestores educacionais e com os gestores das incubadoras, parques tecnológicos e NITTs.

Além das lições aprendidas, o processo de implantação apresentado igualmente pode ser caracterizado como contribuição por relatar experiências úteis que possam ser aproveitadas por instituições que pretendem implantar modelos semelhantes.

As contribuições apresentadas servem, principalmente, para os gestores de ambientes de inovação que trabalham em rede, mas, ao mesmo tempo, podem ser úteis para habitats de inovação que não trabalham em formato de rede.

O processo de implantação da rede ULBRATECH ainda está em processo de amadurecimento, e, em algumas unidades de ensino da ULBRA, falta iniciar projetos de inovação na rede.

Uma das próximas etapas a ser desenvolvida e implantada é a definição de indicadores de maturidade para os habitats de inovação da rede ULBRATECH, que poderá funcionar como um instrumento auxiliar no monitoramento e planejamento de ações de desenvolvimento das incubadoras e parques tecnológicos, assim como os NITTs que compõem a Rede ULBRA de Inovação.

\title{
ULBRATECH: CHALLENGES AND BENEFITS IN DEPLOYING A INNOVATION NETWORK
}

\begin{abstract}
This article presents the experience of developing a Innovation Network, "ULBRATECH Innovation Network" in the form of experiences and lessons learned, obtained through the planning and implementation of this network comprising business incubators, technology parks and centers or agencies of innovation and technology transfer, nationwide in Brazil. This network is organized into fifteen Brazilian cities, comprising six states of the South, Midwest and northern of Brazil, where the university operates with higher education units. We performed an exploratory qualitative research with the managers of innovative companies and managers of the educational units of the university, where they identified the main challenges and difficulties of structuring the network, as well as the main advantages obtained by this network. The results are presented in the form of lessons learned and serve as a source of knowledge for institutions that want to deploy innovation networks. Among the main results, highlights the difficulty of integrating management model of the university with the model of innovation projects, due to the regional diversity, knowledge management issues that impact the integration between network participants, both due to the lack of sharing of experiences, such as the difficulty of maintaining an updated directory of network skills, lack of mechanisms to make better use of opportunities at different points of the network; need to promote entrepreneurial culture both internally and externally to the university, and the need for revision management process of the network, in order to qualify the governance model of the Innovation Network.
\end{abstract}

Keywords: Innovation Networks. Technology Parks. Technology Incubators.

\section{REFERÊNCIAS}

ASSOCIAÇÃO NACIONAL DE ENTIDADES PROMOTORAS DE EMPREENDIMENTOS DE TECNOLOGIAS AVANÇADAS (ANPROTEC). Disponível em: < http://anprotec.org.br/site/pt/incubadoras-e-parques/>. Acesso em: 30 ago. 2013.

CLARK, B. Sustaining Change in Universities. Maidenhead: Open University Press, 2004.

DU PLESSIS, M. The role of knowledge management in innovation. Journal of Knowledge Management, Bringley, v. 11, n. 4, p. 20-29, 2007. 
GLOOR, P. Swarm Creativity: Competitive Advantage Through Collaborative Innovation Networks. Oxford: Oxford University Press, 2005.

HACKETT, S. M.; DILTS, D. M. A Systematic Review of Business Incubation Research. Journal of Technology Transfer, Berlin, v. 29, n. 1, p. 55-82, Jan. 2004.

LIPPARINI, A; SOBRERO, M. Coordinating multi-firm innovative processes: entrepreneur as catalyst in small-firm networks in the formation of inter-organizational networks. Oxford: Oxford University Press, 1997.

MEDEIROS, J. A. et al. Pólos, Parques e Incubadoras: a busca da modernização e competitividade. São Paulo: CNPq, SENAI, SEBRAE, 1992.

MICHEL, M. H. Metodologia e pesquisa em ciências sociais. São Paulo: Atlas, 2009.

NATIONAL BUSINESS INCUBATOR ASSOCIATION (NBIA) What is Business Incubation? Disponível em: <http://www.nbia.org/resource_library/what_is/index.php>. Acesso em: 30 ago. 2013.

PELLEGRIN, I. et al. M. Redes de inovação: construção e gestão da cooperação pró-inovação. Revista de Administração da Universidade de São Paulo, São Paulo, v. 42, n. 3, jul./set. 2007.

UNITED KINGDOM SCIENCE PARK ASSOCIATION (UKSPA). Disponível em: <http://www.ukspa.org.uk>. Acesso em: 30 ago. 2013.

VEDOVELLO, C. Aspectos relevantes de parques tecnológicos e incubadoras de empresas. Revista do BNDES, São Paulo, v. 7, n.14, p. 273-300, dez. 2000.

VEDOVELLO, C.; JUDICE, V.; MACULAN, A. D. Revisão crítica às abordagens a parques tecnológicos: alternativas interpretativas as experiências brasileiras recentes. Revista de Administração e Inovação, São Paulo, v. 3, n. 2, p. 103-118, abr./jun. 2006.

YIN, R. K. Estudo de caso: planejamento e métodos. Porto Alegre: Bookman, 2010. 